\title{
Blood Flow Velocity Waveforms of the Fetal Middle Cerebral Artery in Pregnancies Complicated by Diabetes Mellitus
}

\author{
JUNJI ISHIMATSU, TOURU MATSUZAKI, MICHIAKI YAKUSHIJI \\ AND TEIJI HAMADA* \\ Department of Obstetrics and Gynecology, Kurume University School of Medicine, \\ Kurume 830 and * Social Insurance Kurume Daiichi Hospital, 21 Kushihara, \\ Kurume 830, Japan
}

Received for publication June 13, 1995

\begin{abstract}
Summary: Fetal blood flow velocity waveforms of the middle cerebral artery were measured by pulsed Doppler ultrasound in 43 pregnant women with diabetes mellitus ( 33 class B, 3 class C, 6 class D and 1 class R). The recordings were performed between 24 and 38 weeks of gestation. A total of 122 recordings were analyzed prior to establishing the resistance index (RI= peak systolic velocity minus diastolic velocity/peak systolic velocity). The mean maternal serum glucose was $124.3 \mathrm{mg} / \mathrm{dl}$ (range: 72.7 to $281.5 \mathrm{mg} / \mathrm{dl}$ ), the mean hemoglobin A1c was 6.03\% (range: 3.3 to $11.0 \%$ ) and the mean fructosamine level was $255.97 \mu \mathrm{mol} / \mathrm{L}$ (range: 205 to $397 \mu \mathrm{mol} / \mathrm{L}$ ). The resistance index did not differ between the fetuses of the diabetic mothers in our study population. Additionally, no significant correlation was noted between RI values and serum glucose levels $(r=0.03)$, hemoglobin A1c levels $(r=0.13)$ or fructosamine levels $(\mathrm{r}=0.04)$ during the period of 26 to 34 gestational weeks. These data indicate that the RI within the fetal cerebral artery is unaffected by the maternal glycemic control when mean serum glucose levels are below $280 \mathrm{mg} / \mathrm{dl}$.
\end{abstract}

Key words: blood flow velocity waveforms - middle cerebral artery pulsed Doppler — diabetic pregnancy - ultrasonography

\section{Introduction}

Doppler ultrasound was first used to record blood velocity waveforms within umbilical vessels by Fitzgerald and Drumm (1977). Several reports have since been published on blood flow velocity waveforms in the fetal circulation using this technique (Reuwer et al. 1984; Trudinger et al. 1985). Intrauterine growth retardation and fetal distress have been reported to be associated with abnormal blood flow velocity waveforms in the fetal ascending aorta (Griffin et al. 1984), the fetal anterior cerebral artery (Arbeille et al. 1987) and the umbilical artery (Reuwer et al. 1984; Erskine and Ritchoe, 1985). However, there have been only a few reports examining blood flow velocity waveforms within the umbilical artery in fetuses of diabetic mothers (Bracero et al. 1986; Olofsson et al. 1987; Landon et al. 1989; Ishimatsu et al. 1991). Furthermore, there has been only one paper examining 
blood flow velocity waveforms in the fetal cerebral arterial circulation during diabetic pregnancies (Salvesen et al. 1993). The purpose of the present study was to determine the relationship between blood flow velocity waveforms in the fetal middle cerebral artery (MCA) and the control of maternal glucose in pregnant women with diabetes mellitus.

\section{Materials and Methods}

A pulsed Doppler system (Aloka SSD 650 and SSD 680) with a carrier frequency of $3.5 \mathrm{MHz}$ was used to determine blood flow velocity in the fetal MCA in 43 women whose pregnancies were complicated by diabetes mellitus ( 33 class B, 3 class $\mathrm{C}, 6$ class $\mathrm{D}$ and 1 class $\mathrm{R}$ ). The gestational age of the patients ranged from 24 to 38 weeks and was confirmed by ultrasound examination during the first trimester of pregnancy. All patients had their deliveries at Kurume University Hospital between June, 1989 and December, 1993. Doppler ultrasonography was performed on patients in a semirecumbent position. The standard plane was first obtained to measure the biparietal diameter. This plane included the thalamus and cavum of the septum pellicidum. Pulsation or color flow of the middle cerebral artery could be seen at the level of the insula. The sample volume was placed over the middle cerebral artery and maximum flow velocity waveforms were recorded. The resistance index ( RI=peak systolic velocity minus end diastolic velocity over peak systolic velocity) was measured using calipers, from a mean of 3-4 cycles. The values were then calculated on a computer.
Mean serum glucose levels were obtained from six serial samples (pre and 2-hour post-prandial). The mean serum glucose level was determined within 3 days, and the hemoglobin A1c and fructosamine concentrations were determined within 7 days prior to the ultrasound examination.

\section{Results}

Mean serum glucose levels, mean hemoglobin A1c levels and mean fructosamine levels were $124.29 \mathrm{mg} / \mathrm{dl}$ (range: 72.7 to $281.5 \mathrm{mg} / \mathrm{dl}$ ), $6.03 \%$ (range: 3.3 to $11.0 \%$ ) and $255.97 \mu \mathrm{mol} / \mathrm{L}$ (range: 205 to $397 \mu \mathrm{mol} / \mathrm{L}$ ), respectively, for the patient group.

A total of 122 velocity waveforms were obtained from 43 patients. The RI MCA values were in the normal range (mean $\pm 1.5 \mathrm{SD}$ ) for 103, high in 14 and low in 5 pregnancies (Fig. 1).

A total of 87 MCA RI recordings were performed with either maternal serum glucose assays (106 samples), hemoglobin A1c assays (67 samples) and/or fructosamine assays (31 samples). At 24-38 weeks of gestation the RI values were scattered and did not correlate with either mean serum glucose levels (Fig. 2), hemoglobin A1c levels (Fig. 3) or fructosamine levels (Fig. 4).

The changes in RI values of the MCA during pregnancy appeared to be stationary between 26 and 34 gestational weeks. Thus, the correlation between these values and the parameters of glycemic control was re-evaluated during this time period. There was no significant correlation between the RI values and serum glucose $(r=0.03)$, 


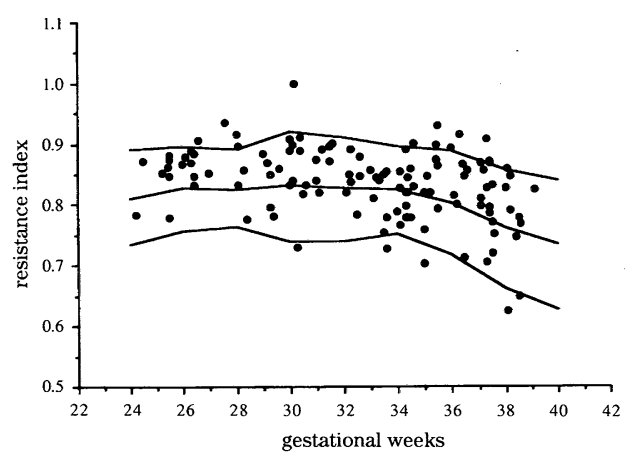

Fig. 1. Resistance indices of the fetal middle cerebral artery during 43 diabetic pregnancies superimposed on the normal values (mean \pm $1.5 \mathrm{SD})$.

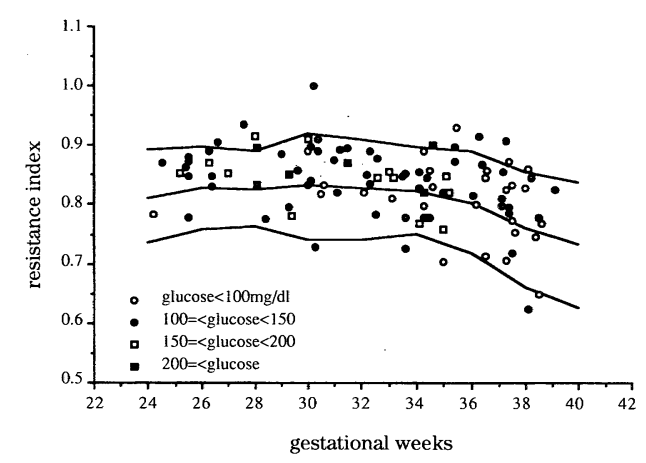

Fig. 2. Resistance indices of the fetal middle cerebral artery during 39 diabetic pregnancies plotted relative to mean maternal serum glucose levels; $\bigcirc$ : glucose $<100 \mathrm{mg} / \mathrm{dl}$, -: glucose, 100 to $149 \mathrm{mg} / \mathrm{dl}, \square$ : glucose, 150 to $199 \mathrm{mg} / \mathrm{dl}$, $\mathbf{\square}$ : glucose $\geqq 200 \mathrm{mg} / \mathrm{dl}$.

hemoglobin Alc ( $\mathrm{r}=0.13)$ or fructosamine levels (0.04).

Fifteen RI recordings were obtained from 7 patients whose infants developed polycythemia. Of these, the RI was high in 3 , normal in 10 and low in 2 . The sizes

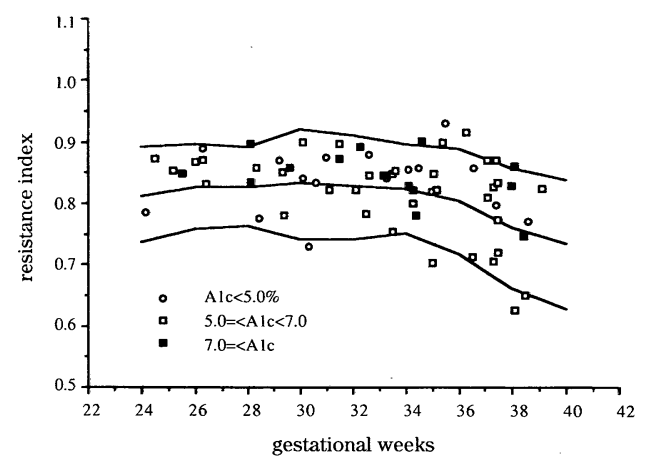

Fig. 3. Resistance indices of the fetal middle cerebral artery during 36 diabetic pregnancies plotted relative to maternal hemoglobin A1c levels; $\bigcirc$ : A1c $<5.0 \%, \square$ : Alc, 5.0 to $6.9 \%, \square: \mathrm{A} 1 \mathrm{c} \geqq 7.0 \%$.

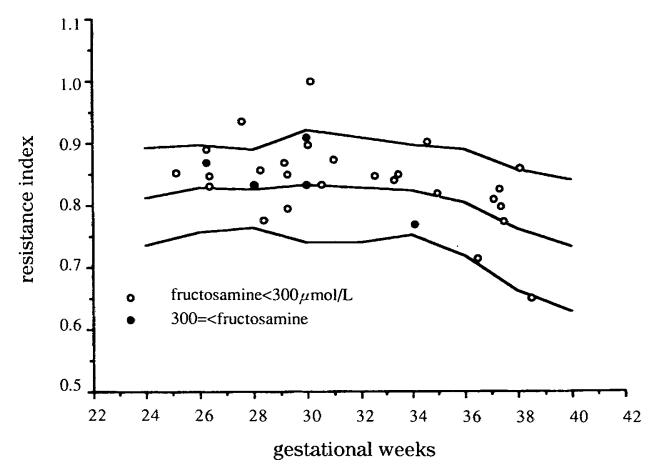

Fig. 4. Resistance indices of the fetal middle cerebral artery during 18 diabetic pregnancies plotted relative to maternal fructosamine levels; $\bigcirc$ : fructosamine $<300 \mathrm{E} \mathrm{mol} / \mathrm{L}$, ๑: fructosamine $\geqq 300 \mu \mathrm{mol} / \mathrm{L}$.

of the infants were appropriate-for-dates in 30 cases, large-for-dates in 12 cases and small-for-dates in one case. There was no evidence of fetal distress in any of the subjects during non-stress testing. 


\section{Discussion}

The Doppler ultrasound method has been increasingly used in obstetrics to measure blood flow velocities of the umbilical and fetal vessels. Normal waveforms always demonstrate forward flow due to the low peripheral circulatory resistance in the fetus and placenta. The $\mathrm{RI}$ in the umbilical artery decreases with advancing gestational age. The RI in the MCA increases at 29 and 30 weeks of gestation and decreases by the end of the pregnancy. Changes in the RI of the cerebral artery appear to be more pronounced than those of the umbilical artery during pregnancy. Intrauterine growth retardation, fetal distress, hypertensive disease or toxemia have been associated with elevations in the pulsatility index, $\mathrm{RI}$ and $\mathrm{S} / \mathrm{D}$ ratio in the umbilical artery (Erskine and Ritchoe, 1985; Trudinger et al. 1985; Wladimiroff et al. 1986), as well as a decrease in the RI of the cerebral artery (Arbeille et al. 1987; Ishimatsu et al. 1989).

Elevated RI values and S/D ratios in the umbilical artery have been reported in diabetic pregnancies with maternal vasculopathy. However, only four reports have examined the relationship between umbilical artery velocity waveforms and maternal blood glucose levels. Bracero et al. (1986) have found a significant positive correlation between $\mathrm{S} / \mathrm{D}$ ratios and maternal serum glucose levels. The pulsatility index of the umbilical artery has been noted to be higher in diabetic relative to non-diabetic pregnant patients, indicating that the placental vascular resistance is elevated in diabetic pregnancies (Olofsson et al. 1987). However, no significant correlation has been observed between third trimester $\mathrm{S} / \mathrm{D}$ ratios and glycosylated hemoglobin or mean blood glucose levels (Landon et al. 1989). It has been previously reported that no significant correlation exists between umbilical artery $R I$ values and serum glucose or fructosamine levels in diabetic pregnancies in the absence of vascular complications (Ishimatsu et al. 1991). However, abnormal umbilical artery velocity waveforms were not found when serum glucose levels were maintained below $200 \mathrm{mg} / \mathrm{dl}$. Poor diabetic control resulting in serum glucose levels above $300 \mathrm{mg} / \mathrm{dl}$ may lead to a rise in umbilical artery $\mathrm{RI}$ values.

Increased blood flow velocity is considered to be mediated by a decreased blood viscosity in anemic fetuses in pregnancies complicated by red blood cell isoimmunization (Nicolaides and Kaminopetros, 1992). In fetuses of diabetic mothers, however, blood viscosity is increased as a consequence of polycythemia (Foley et al. 1981). In the present study, all MCA RI values were normal in the 6 patients with maternal serum glucose levels above $200 \mathrm{mg} / \mathrm{dl}$. There were no characteristic RI findings in the cases complicated by polycythemia during the neonatal period. None of the patients had vascular complications and no significant correlation was found between the RIs and serum glucose, hemoglobin Alc or fructosamine. The majority of RI values for the MCA in diabetic pregnancies were within the normal range for pregnant patients. These data suggest that the fetal MCA RI is not affected by maternal glycemic control when serum glucose levels range from 73 to $282 \mathrm{mg} / \mathrm{dl}$, hemoglobin A1c levels range from 3.3 to $11.2 \%$ and fructosamine levels range from 210 
to $397 \mu \mathrm{mol} / \mathrm{L}$. Salvesen et al. (1993) have reported that the mean velocities of the fetal descending thoracic aorta and the middle cerebral artery were normal with no significant association between the degree of fetal polycythemia and the Doppler indices. Furthermore in pregnancies complicated by diabetes mellitus, fetal acidemia and polycythemia, Doppler studies indicated that the placental and fetal circulations remained essentially normal with the exception of patients complicated by IUGR or eclampsia.

The current and previous results (Ishimatsu et al. 1991) suggest that as long as the maternal serum glucose level is maintained below $200 \mathrm{mg} / \mathrm{dl}$ and the fetus is not exposed to chronic hypoxia or intra-uterine growth retardation, increases in fetal blood viscosity are mild and normal cerebral and placental circulation can be maintained. The RI values for the fetal MCA and the umbilical artery are not affected by maternal glycemic control during pregnancies complicated by diabetes mellitus in the absence of maternal vasculopathy.

\section{References}

Arbeille, P., Roncin, A., Berson, M., Patat, F. and Pourcelot, L. (1987). Exploration of the fetal cerebral blood flow by duplex Doppler linear array system in normal and pathological pregnancy. Ultrasound in Med. and Biol. 13, 329-336.

Bracero, L., Schulman, H., Fleischer, A., Farmakides, G. and Rochelson, B. (1986). Umbilical artery velocimetry in diabetes and pregnancy. Obstet. Gynecol. 68, 654-658.

ERskine, R.L.A. and RITCHOE, J.W.K. (1985).
Umbilical artery blood flow characteristics in normal and growth-retarded fetuses. Br. J. Obstet. Gynecol. 92, 605-610.

Fitzgerald, D.E. and Drumm, J.E. (1977). Non invasive measurement of human fetal circulation using ultrasound. A new method. Br. Med. J. 3, 1450-1451.

Foley, M.E., Collins, R., Stronge, J.M., Durry, M.I. and Macdonald, D. (1981). Blood viscocity in umbilical cord from babies of diabetic mother. J. Obstet. Gynecol. 2, 93-96.

Griffin, D., Bilardo, D., Mashini, L., Diazrecasens, J., Pearce, J.M. et al. (1984). Doppler blood flow waveforms in the descending thoracic aorta of the human fetus. Br. J. Obstet. Gynecol. 91, 997-1006.

Ishimatsu, J., Hotta, M., Matsunaga, T., Manabe, A.,Yoshimura, O. et al. (1989). Cerebral artery blood flow velocity waveforms in normal and small for date fetuses. Kurume Med. J. 36, 181-184.

Ishimatsu, J., Yoshimura, O., Hotta, M., Matsunaga, T., Matsuzaki, T. et al. (1991). Umbilical artery blood flow velocity waveforms in pregnancy complicated by diabetes mellitus. Arch. Gynecol. Obstet. 248, 123-127.

Landon, M.B., GabBe, S.G., Bruner, J.P. and LuDMIR, J. (1989). Doppler umbilical artery velocimetry in pregnancy complicated by insulin-dependent diabetes mellitus. Obstet. Gynecol. 73, 961-965.

Nicolaides, K.H. and Kaminopetros, P. (1992). The clinical value of waveforms from the descending thoracic aorta: red cell iso-immunization. In Doppler ultrasound in perinatal medicine, ed. Pearce, J. M., pp. 244-257. Oxford: Oxford University Press.

Olofsson, P., Lingman, G., Marsal, K. and SJoberg, N.O. (1987). Fetal blood flow in diabetic pregnancy. J. Perinat. Med. 15, 545-553.

Reuwer, P.J.H.M., Bruinse, H.W., Stouten, P. and Haspels, A.A. (1984). Doppler assessment of the fetoplacental circulation in the normal and growth retarded fetuses. Europ. J. Obstet. Gynecol. Reprod. Biol. 18, 199-205.

Salvesen, D.R., Higueras, M.T., Mansur, C.A., Freeman, J., Brudenell, M. et al. (1993). 
Placental and fetal Doppler velocimetry in pregnancies complicated by maternal diabetes mellitus. Am. J. Obstet. Gynecol. 168, 645-652.

Trudinger, B.J., Сook, C.M. and Giles, W.B. (1985). Fetal umbilical artery flow velocity waveforms and placental resistance: clinical significance. Br. J. Obstet. Gynecol. 92, 23-30.

Wladimiroff, J., Tonge, H.M. and Stewart, P.A. (1986). Doppler ultrasound assessment of cerebral blood flow in the human fetus. Br. J. Obstet. Gynecol. 93, 471-475. 